\title{
La satisfacción de los clientes del proyecto Cantera de héroes envigado fútbol club
}

\author{
Costumers satisfaction of cantera de héroes envigado fútbol club \\ project
}

\begin{abstract}
Andrés Leonardo Colorado-Arango ${ }^{1}$ M.Sc, Erwin Alexis Cardozo-Molina² Lic.
${ }^{1}$ Universidad de Antioquia - Politécnico Colombiano Jaime Isaza Cadavid, Universidad de San Buenaventura. Grupo de Estudios Interdisciplinarios en Educación ESINED. Magíster en Administración, Licenciado en Educación Física. ${ }^{2}$ Universidad de San Buenaventura. Auxiliar de investigación en Grupo de Estudios Interdisciplinarios en Educación ESINED. Cartagena, Colombia. ${ }^{*}$ Correspondencia: andreslc24@gmail.com.
\end{abstract}

Recibido: 28-02-2017; Aceptado: 18-04-2017

\section{RESUMEN}

Esste estudio tuvo como objetivo evaluar el nivel de satisfacción de los clientes del proyecto Cantera de Héroes Envigado Fútbol Club durante el año 2016, partiendo de un rastreo conceptual de la expresión "satisfacción del cliente", la indagación de antecedentes investigativos y finalmente, la comprobación empírica de dicho constructo con la aplicación de la "Escala de Percepción de Organizaciones Deportivas" (EPOD), construida por Nuviala Nuviala, Tamayo Fajardo, Iranzo Llopis y Falcón Miguel (2008), la cual evalúa la satisfacción en cuatro dimensiones del servicio como son las de técnicos, actividades, instalaciones y comunicación. El total de la población estudiada fue un censo de los 140 padres de familia con hijos en el programa. Las mejores valoraciones recayeron en las dimensiones de técnicos y actividades.

Palabras clave: Satisfacción, servicio, clientes, calidad

\section{ABSTRACT}

The objective of this study was to evaluate the level of customer satisfaction of the Cantera de Héroes Envigado Fútbol Club project in 2016, based on a conceptual tracing of the expression customer satisfaction, the investigation of investigative antecedents and finally the empirical verification of said construct with the application of the "perception scale of sports organizations" (EPOD), built by Nuviala Nuviala, Tamayo Fajardo, Iranzo Llopis and Falcón Miguel (2008), which evaluates satisfaction in four dimensions of the service such as technicians, Activities, facilities and communication. The total population studied was a census of 140 parents with children in the program. The best evaluations fell on the size of technicians and activities.

Keywords: Satisfaction, service, customers, quality 


\section{INTRODUCCIÓN}

El programa de iniciación deportiva denominado Cantera de Héroes Envigado Fútbol Club abrió sus puertas a finales del año 2014, para dar respuesta a una demanda de padres de familia interesados en procesos deportivos a temprana edad. Lo más llamativo ocurrió durante el año 2015 al producirse un crecimiento exponencial en la cantidad de niños participantes; esta situación generó un interés por conocer las causas de dicho fenómeno, en la comunidad investigativa de la administración deportiva, planteándose en principio estudiar la variable relacionada con la satisfacción de los clientes.

Este trabajo pretende propiciarle a los niños alcanzar su máximo potencial motor y fortalecer el vínculo con sus padres, utilizando el fenómeno del fútbol como un elemento capaz de generar una gran motivación entre sus practicantes (niños, jóvenes y adultos), utilizando como estrategia el aprendizaje del juego por los infantes, a través de la imitación, pues en la primera infancia se aprovecha la etapa de aprendizaje motor sensible; por tal razón, es importante el acompañamiento de los padres en cada una de las clases.

La aceptación experimentada por este proyecto por parte de la academia, los padres de familia y el sector deporte en general, lleva a pensar en la existencia de elementos particulares en la propuesta, porque en Colombia, el saber relacionado con el trabajo en el campo de la iniciación deportiva en la primera infancia, aún es incipiente frente a producción científica, que sirva como referente para la construcción de ideas pertinentes para la población entre cero y seis años, considerando la existencia de una gran oferta de programas deportivos en los cuales, se asume como usuario a esa población,. Por ello, la academia debe preocuparse por investigar esa situación y encontrar evidencias acerca del comportamiento de las variables apuntadas en este proyecto y de esta forma develar los aspectos administrativos vinculados a su éxito.

Desde la óptica del autor se evidencia una gran oportunidad para identificar los aspectos que propiciaron el auge en las inscripciones del programa, ya que, esta información se convertirá en material de consulta y validación para el diseño de propuestas deportivas que intervengan con una población similar, además de posibilitar a los administradores actuales de la escuela de iniciación, el análisis del servicio para tomar las decisiones necesarias con miras a la consolidación de la oferta actual.

Como consecuencia de lo expresado, se elaboró un diseño de investigación, orientado a determinar la evolución del concepto de "satisfacción del cliente" hasta la actualidad, la construcción de un marco de antecedentes de investigación sobre el estudio de la satisfacción en el campo de los servicios deportivos por último, emplear un instrumento con validez científica y capaz de generar información cuantificable de las diferentes dimensiones involucradas en los servicios en este campo, con el objetivo de describir cuales son los elementos diferenciadores del proyecto "Cantera de Héroes" y el perfil sociodemográfico de los clientes, para aportar a la gestión y planeación de propuestas de servicios deportivos en la primera infancia.

Luego de realizar una amplia revisión de los antecedentes de investigación en el campo de los servicios deportivos como: Arocas y Mundina (1998), Nuviala y Casajús (2005), Molina y Vargas (2008), Crespo y Pérez-Campos (2011), Elasri, Triadó, y Aparicio (2013), Elasri, Triadó, y Chueca (2015) e Inderenvigado (2015); se tomó la decisión de emplear como instrumento de evaluación de la satisfacción de 
los clientes del proyecto "Cantera de Héroes", el propuesto por Nuviala, Tamayo, Iranzo y Falcón (2008), en el artículo "Creación, diseño, validación y puesta en práctica de un instrumento de medición de la satisfacción de usuarios de organizaciones que prestan servicios deportivos". Ese trabajo consistió en construir una escala de medición de la calidad del servicio prestado por una organización desde la perspectiva de los usuarios, además, analizaron la percepción del centro deportivo donde realizan sus prácticas.

Se empleó un instrumento para medir la fiabilidad(CoeficienteAlphadeCronbachCAC ${ }^{1}$ y una técnica en el trabajo de campo (cuestionario auto administrado con la presencia del encuestador con el fin de mejorar la comprensión de todos los ítems, mientras se incrementan las propiedades psicométricas del mismo). La investigación de la escala de 28 ítems arrojó como resultado un CAC $=0,919$, siendo este una excelente calificación y un firme argumento para tomarlo como referente para la investigación.

\section{METODOLOGÍA}

Esta investigación se desarrolló con un enfoque mixto, utilizándose herramientas cualitativas (revisión documental para la construcción de los marcos conceptuales y los antecedentes por medio de consulta en bases de datos bibliográficas), y un proceso de nivel descriptivo de corte transversal-cuantitativo, en el cual se aplicó el cuestionario sugerido por Nuviala y otros (2008), para evaluar la satisfacción de los usuarios del proyecto en mención, en las dimensiones de entrenadores, instalaciones deportivas, actividades desarrolladas y la imagen de una organización por medio de 28 ítems, además de la identificación del

\footnotetext{
$\begin{array}{lll}1 & \text { Según Nuviala, Tamayo, Iranzo y Falcón (2008), uno de los coeficientes más }\end{array}$ interna de una prueba y refleja el grado en que convocarían las preguntas de la escala, su valor

puede variar entre cero y uno.
George y Mallery (1995), citados por Nuviala, Tamayo Fajardo, Iranzo y Falcón (2008), propusieron la siguiente valoraciones para Alpha: > 0,9-Excelente; 0,9-0,8-Bueno; 0,8-0,7-Aceptable; 0,7-0,6-Débil; 0,6-0,5-Pobre; y <0,5- No aceptable.

perfil sociodemográfico de los clientes del proyecto.

Se planteó como población objeto de la investigación el $100 \%$ de los clientes del proyecto, con el interés de obtener resultados lo más veraces posible, por lo cual se aplicaron 140 cuestionarios a los padres de familia con hijos en el proyecto, con la presencia del encuestador, buscando mejorar la comprensión de todos los ítems, e incrementar así, la seguridad frente al correcto diligenciamiento del mismo, durante los días 20, 22, 24 y 25 de septiembre de 2016 en los horarios de entrenamiento: martes y jueves a las 5:00 p.m. y sábados y domingos de 8:00 am, 9:00 a.m. y 10:00 a.m. ; .

Anexo 1. Encuesta de satisfacción del usuario

Para el análisis descriptivo de las respuestas del cuestionario, se utilizó el paquete estadístico SPSS versión 24 y el Alpha de Crombach (parámetro de validez de cada dimensión y del cuestionario en general).

\section{RESULTADOS}

Marco conceptual. En cumplimiento de uno de los objetivos de la investigación, se construyó el siguiente análisis del constructo "satisfacción del consumidor". Para comenzar, varios autores estudiaron la satisfacción del consumidor desde diferentes disciplinas (Arocas y Mundina, 1998). Según Tse, Nicosia y Wilton (1990), la satisfacción ha sido utilizada por los economistas para indicar la efectividad del sistema de marketing (Garner, 1981): los sociólogos, reflejan el bienestar social, (Campbell, 1976); los psicólogos, indican el sentimiento emocional de los individuos, (Quintanilla, 1994; Rubenstein, 1982) y por los políticos, subrayan los problemas del mercado, (Scherer, 1980). 
Arocas y Mundina (1998), desde sus análisis definen la satisfacción del consumidor como un proceso subjetivo multidimensional y dinámico, donde le dan importancia a la experiencia del antes, durante y después de realizada la compra. Complementan lo expresado por Arocas y Mundina (1992), citado por Martínez y Martínez (2010), ellos consideran la satisfacción del consumidor como una actitud; una evaluación subjetiva sobre las experiencias de consumo. Así, ese concepto es entendido como un juicio evaluativo realizado por el consumidor de forma global, sobre su experiencia de consumo en un momento posterior a él.

Varios autores citados por Crespo y Pérez-Campos (2011), al estudiar la expresión "satisfacción del consumidor" expresaron que también aparece en la literatura relacionada con la facilitación de conductas futuras (Oliver y Swan, 1989), la disminución de las quejas y reclamaciones y el incremento de la lealtad (Fornell, Johnson, Anderson, Cha y Bryant, 1996), con fenómenos post-compra tales como: cambios actitudinales, re-compra y fidelidad a la marca (Churchill y Surprenant, 1982), - la disposición a pagar (Homburg, Koschate y Hoyer, 2005). Ottar (2002) señaló: "...la satisfacción deriva de la calidad y afecta directamente a la fidelidad en la recompra". Otros investigadores citados por Elasri, Triadó y Aparicio (2013), como Oliver (1980), definieron "la satisfacción del cliente" como una sorpresa inherente a la experiencia de compra, de finita duración y luego, decae, en actitudes hacia la compra efectuada.

Con la misma línea de pensamiento, ese asunto también se examinó por Tse y Wilton (1988), quienes conceptualizaron la satisfacción como la evaluación ejercida por el sujeto al contraponer las expectativas creadas con antelación y el resultado obtenido del consumo de un producto o servicio. Luego Cronin y Taylor (1992) y Parasuraman et al. (1988), valoraron la diferenciación entre los conceptos de calidad del servicio percibido y satisfacción, y consideraron al primero como una actitud duradera a lo largo del tiempo, y al segundo como un juicio transitorio ante un servicio específico.

Marco de antecedentes. En cumplimiento de otro de los objetivos de esta investigación, se rastreó la influencia de trabajos españoles como antecedentes sobre la satisfacción en el campo de los servicios deportivos.

En Valencia (España), Arocas y Mundina (1998), en el artículo: La satisfacción del consumidor en el marketing del deporte, determinaron la gestión deportiva de una escuela de vela pública de la comunidad valenciana. En ese trabajo se utilizó el cuestionario "Neptuno-1", (mide entre otras variables relacionadas con el deporte náutico, la satisfacción de los usuarios en su práctica). Esta investigación presenta los resultados de 10 variables. La satisfacción general con la escuela de vela fue $34,8 \%$.

Luego, en Huelva (España), Nuviala y Casajús (2005), en el artículo: "Calidad percibida del servicio deportivo en edad escolar desde la perspectiva de los padres", desarrollaron una investigación, cuyo objetivo era conocer el grado de satisfacción de los padres con hijos participantes en programas públicos de actividades deportivas en edad escolar, con relación a los recursos humanos y materiales. El instrumento utilizado fue un cuestionario auto-administrado con presencia del encuestador. Los resultados se analizaron con el paquete estadístico SPSS 12.0, donde en general, los progenitores estuvieron muy satisfechos con el técnico deportivo de sus hijos, además de expresar algunas inconformidades frente a los recursos materiales empleados en las sesiones de entrenamiento. 
Los profesores Nuviala, Tamayo, Iranzo y Falcón (2008), publicaron el artículo, "Creación, diseño, validación y puesta en práctica de un instrumento de medición de la satisfacción de usuarios de organizaciones que prestan servicios deportivos", dicho trabajo, consistió en construir una escala para medir la calidad del servicio prestado por una organización desde la perspectiva de los usuarios, y permite el análisis de la percepción del centro deportivo donde se desarrollan sus prácticas. Se aplicó un cuestionario auto-administrado de 28 ítems, el cual se validó con un Alpha de Cronbach=0,919.

En el 2011 Crespo y Pérez-Campos, publicaron el artículo denominado: "La influencia de la satisfacción con la programación de partidos en la intensión de asistir de los espectadores", planteándose como principal objetivo el identificar el grado de satisfacción de los espectadores con la programación de los partidos y su relación con las intenciones futuras. La técnica utilizada fue el cuestionario; se administró en las salidas de la instalación deportiva al finalizar la competición y se solicitó responder los ítems y lo devolvieran en los siguientes partidos en las mesas de recogida. Los espectadores valoraron la satisfacción general con una media de 5,47 y las intenciones futuras con un 6,19 . En concreto, valoraron, las intenciones de seguir asistiendo, con una media de 6,27 y la intención de recomendar la asistencia a otros posibles espectadores con 6,06, lo anterior teniendo en cuenta que se utilizó una escala de 1 a 10.

En Barcelona (García, Cepeda y Martín, 2012), en su artículo: "La satisfacción de clientes y su relación con la percepción de calidad en centro de fitness" aplicaron la escala CALIDFIT, aportando una medida para evaluar la percepción de la calidad de los clientes de centros de fitness privados (CFP), segmentándola en niveles, además, comprobaron su influencia en la satisfacción. La recogida de datos se llevó a cabo mediante un cuestionario (instrumento elaborado a partir de las medidas propuestas por Parasuraman et al. (1988), Sanz et al. (2005), Calabuig et al. (2008) y Nuviala et al. (2008). Los resultados demuestran la percepción de calidad de los usuarios con una escala fiable y válida de Alfa de Cronbach $=0.916$.

El subgrupo de los hombres (25 a 34 años) con ocupación laboral pública percibió con menor calidad todas las dimensiones obtenidas. En el lado contrario, las mujeres jubiladas (55 a 65 años) apreciaron mayor calidad en el personal y los servicios.

En Sevilla, Nuviala Nuviala et al (2012), publicó el articulo denominado "Calidad, satisfacción y valor percibido de los usuarios de un servicio deportivo público", cuyo objetivo fue evaluar y establecer modelos de predicción de la calidad observada, satisfacción y valor percibido de los usuarios de un servicio deportivo. Para la obtención de datos se utilizó el cuestionario EPOD2 (Nuviala et al, en prensa) compuesto por un total de 25 ítems de respuesta alternativa Likert, con una oscilación entre 1 (muy en desacuerdo) y 5 (muy de acuerdo), con distintas áreas de evaluación: 1. Calidad percibida (20 ítems), 2. Satisfacción (4 ítems) y 3 . Valor del servicio (1 ítem). La valoración media de la calidad percibida obtenida con la suma de ítems de la escala fue buena: $3.80 \pm .61$, sobre un máximo de 5 .

La dimensión "técnicos" obtuvo la evaluación más alta por parte de los usuarios, le siguió la del personal de servicios. Por su parte, la de "comunicación" y "material" resultaron ser las de peores calificaciones. La satisfacción de los usuarios fue muy buena (4.14t.76), no existiendo diferencias significativas entre los grupos de actividades. El valor percibido del servicio logró una puntuación media de 3.89 .94 . El fitness, en general, alcanzó la valoración más alta. 
Elasri, Triadó y Aparicio (2013) publicaron en Barcelona, el artículo "Evolución de los factores de satisfacción de clientes en centros deportivos entre 1996 y 2013. ¿Cómo han cambiado las percepciones de los usuarios?", donde analizaron los elementos con mayores influencias en la satisfacción de los clientes en los centros deportivos de esa ciudad.

En este estudio, en una primera aproximación se aplicó un pre-test a alumnos y exalumnos de las ediciones 2010-2011, 2011-2012 y 2012-2013, del Máster Oficial en Dirección de Empresas del Deporte y del Máster en Gestión Económica de Entidades Deportivas. Se trata de un alumnado muy ligado al sector objeto de estudio. Los resultados fueron positivos (la media de los clientes manifiesta un nivel de satisfacción de 7,39 puntos, en una escala del 1 al 10). En particular, los aspectos mejor valorados por los clientes son la limpieza y las instalaciones en su conjunto, con una media de 7,92 y 7,75 puntos en el mismo orden; con respecto a hacer amigos en el centro, se sintieron menos satisfechos, siendo la puntuación de solo 4,91.

Otra vez, Elasri, Triadó y Aparicio (2015), presentaron la investigación denominada: "La satisfacción de los clientes de los centros deportivos municipales de Barcelona". En esta identifican y analizan las variables con mayores influencias en la satisfacción del cliente en los centros deportivos estudiados. Para obtener los datos aplicaron una encuesta con cinco dimensiones, que contiene los múltiples componentes de la oferta de servicios en dichas instalaciones: su calidad, los recursos humanos, la comunicación, el entorno social y la relación calidadprecio.

La media de los clientes actuales de los centros deportivos municipales de Barcelona, manifestó un nivel de satisfacción elevado ( 7,83 puntos), en una escala del 1 al 10 . En particular, los aspectos mejor valorados fueron los relacionados con los recursos humanos (el trato ofrecido por parte de los profesionales técnicos $(8,33)$ y del personal auxiliar $(7,89)$, y su nivel formativo $(7,98)$. El aspecto de menos satisfacción resultó ser el de las relaciones sociales en el centro, situándose su único indicador por debajo de los cinco puntos.

En la revisión de antecedentes investigativos nacionales se encontró muy poca producción en el contexto de la satisfacción de los servicios deportivos, pues solo se ubicaron los trabajos de, en Medellín, con la monografía de especialización en Administración Deportiva nombrada: "Evaluación de la satisfacción de los clientes de la liga antioqueña de fútbol con respecto a los programas o servicios durante el primer semestre del 2007, con el propósito de contribuir a mejorar su calidad". Ellos aplicaron encuestas dirigidas a los diferentes tipos de usuarios, sobre la base de un muestreo aleatorio. El procesamiento de la información se efectuó utilizando el software SSPS versión 11.5 .

Henao y Vargas (2008), como fortalezas de la entidad identificaron: la calidad en los contenidos de los programas de formación, el conocimiento de los instructores, el buen trato y atención brindada por los empleados de la LIGA. Por último, el ejercicio realizado por el INDER Envigado en el año2015, donde se indagó por el nivel de satisfacción de los usuarios de los cuatro componentes (deporte, recreación, educación física y actividad física) que ofrece el INDER Envigado, a partir de la evaluación de los ítems, a saber: infraestructura, servicios, talento humano y medios de comunicación; se utilizaron los métodos recolección de información y aplicación de encuestas (dirigidas a los usuarios). EI INDER Envigado, se calificó de la siguiente manera: 
Revista Búsqueda - Enero/Junio de 2017

\begin{tabular}{llcc}
\hline \multicolumn{1}{c}{ Ítems } & \multicolumn{2}{c}{ Calificaciones } & Porcentaje (\%) \\
\hline 1 & Infraestructura & Regular & 43.68 \\
2 & Servicios prestados & Bueno & 42.82 \\
& $\begin{array}{l}\text { Sistema de información y medios } \\
\text { de difusión }\end{array}$ & Regular & 45.23 \\
4 & Talento humano & Excelente & 48.85 \\
\hline
\end{tabular}

Perfil social. Luego de la tabulación y análisis de los datos recolectados, se obtuvieron los siguientes datos sociodemográficos de los padres de familia (clientes del proyecto Cantera de Héroes del Envigado FC) (Tablas 1-5).

Tabla 1. Género del padre o de la madre

\begin{tabular}{ccc}
\hline Género & Frecuencia & $\%$ \\
\hline Masculino & 79 & 56,4 \\
Femenino & 61 & 43,6 \\
\hline Total & 140 & 100,0 \\
\hline Elaboración propia en SPSS 24 & &
\end{tabular}

Tabla 2. Estado civil del padre o de la madre

\begin{tabular}{ccc}
\hline Estado civil & Frecuencia & \% \\
\hline Casado/a & 74 & 52,9 \\
Unión libre & 36 & 25,7 \\
Soltero/a & 27 & 19,3 \\
Divorciado/a & 2 & 1,4 \\
Separado/a & 1 & 0,7 \\
\hline Total & 140 & 100,0 \\
\hline
\end{tabular}

Elaboración propia en SPSS 24

Tabla 3. Lugar de residencia

\begin{tabular}{cccc}
\hline & Lugares & Frecuencia & \% \\
\hline Envigado & 56 & 40,0 \\
Medellín & 49 & 35,0 \\
Itagüí & 14 & 10,0 \\
La Estrella & 10 & 7,1 \\
Sabaneta & 7 & 5,0 \\
& Bello & 3 & 2,1 \\
& Caldas & 1 &, 7 \\
\hline Total & & 140 & 100,0
\end{tabular}

Elaboración propia en SPSS 24

Tabla 4. Edad del padre o de la madre

\begin{tabular}{ccc}
\hline Edad & Frecuencia & \% \\
\hline $30-45$ & 81 & 57,9 \\
$25-30$ & 28 & 20,0 \\
$45-60$ & 11 & 7,9 \\
Menor de 18 & 10 & 7,1 \\
$18-25$ & 10 & 7,1 \\
\hline Total & 140 & 100,0 \\
\hline
\end{tabular}

Elaboración propia en SPSS 24
Tabla 5. Nivel educativo del padre o de la madre

\begin{tabular}{ccc}
\hline Nivel educativo & Frecuencia & $\%$ \\
\hline Profesional & 47 & 33,6 \\
Posgrado & 32 & 22,9 \\
Bachiller & 28 & 20,0 \\
Técnico & 21 & 15,0 \\
Tecnólogo & 10 & 7,1 \\
Primaria & 2 & 1,4 \\
\hline Total & 140 & 100,0 \\
\hline
\end{tabular}

Elaboración propia en SPSS 24

Los acompañantes de los niños incorporados al programa según la distribución de género, son masculinos $(56,4 \%)$ (Tabla 1$)$; el estado civil de los encuestados el $52,9 \%$ es casado/a; el $25,7 \%$ en unión libre, el tercer mejor valorado es $19,3 \%$ soltero/a (tabla 2 ). En cuanto al lugar de residencia de los padres o acompañantes de los niños, el municipio de Envigado fue el mayor representado con un 40,0\%, dato apenas entendible, en la medida que la escuela queda ubicada en este municipio: le siguen en orden de importancia, Medellín (35,0\%) (Tabla 3).

En relación con la edad de los encuestados, el porcentaje mayor $57,9 \%$, lo obtuvieron las personas de 30-45 años, seguidos del grupo de 2530 años (20,0\%) (Tabla 4). De acuerdo con el nivel educativo de la población, los resultados son muy variables: el $33,6 \%$ de las personas encuestadas tienen estudios universitarios, seguidos de aquellos con educación superior y con superación de posgrado (22,9\%); estos datos muestran a una proporción de los acompañantes con altos niveles de formación (56.5\%); le siguen quienes cursaron estudios técnicos $(15,0 \%)$ y los tecnólogos $(7,1 \%)$ (Tabla 5 ). Resultados cuestionario de
satisfacción. La satisfacción de los
usuarios con relación a la dimensión
"técnico", muestra una aceptación muy
positiva de los padres o acompañantes
con respecto a las diferentes actividades
llevadas cabo en las sesiones de
entrenamiento con una $\bar{X}=4,78$ (Tabla
7). Un aspecto evaluado que reafirma
lo anterior es, el profesor es respetuoso 
Colorado-Arango y Cardozo-Molina - La Satisfacción de los clientes del proyecto Cantera de héroes

con una puntuación de 4,91 y, el aspecto con menor valor es el profesor adapta las clases a las necesidades de los usuarios con un 4,71, siendo esta calificación positiva (Tabla 8).

Tabla 7. Estadístico descriptivo

\begin{tabular}{|c|c|c|c|c|c|}
\hline \multirow{2}{*}{$\begin{array}{l}\text { Estadístico } \\
\text { descriptivo }\end{array}$} & \multirow{2}{*}{$\mathbf{N}$} & \multicolumn{2}{|c|}{ Valores } & \multirow{2}{*}{$\bar{X}$} & \multirow{2}{*}{$\mathbf{s}$} \\
\hline & & Mínimo & Máximo & & \\
\hline Dimensión técnica & 140 & 3,17 & 5,00 & 4,7833 & ,36190 \\
\hline$N$ válido (por lista) & 140 & & & & \\
\hline
\end{tabular}

Leyenda: N-Número de muestra; S- Desviación estándar; $\bar{X}$-Media aritmética

Nota: Adaptación de dimensiones de la percepción (Nuviala et al, 2008, pp. 11). Elaboración propia en SPSS 24

Tabla 8. Dimensión técnicos

\begin{tabular}{|c|c|c|c|c|c|}
\hline \multirow{2}{*}{ Dimensión técnicos } & \multirow{2}{*}{$\mathrm{N}$} & \multicolumn{2}{|c|}{ Valores } & \multirow{2}{*}{$\bar{X}$} & \multirow{2}{*}{$\mathrm{S}$} \\
\hline & & Mínimo & Máximo & & \\
\hline Profesor es respetuoso & 140 & 3 & 5 & 4,91 & ,337 \\
\hline $\begin{array}{l}\text { Está contento/a con el trato de } \\
\text { los profesores }\end{array}$ & 140 & 2 & 5 & 4,81 & ,463 \\
\hline $\begin{array}{l}\text { Presta el profesor una atención } \\
\text { adecuada a los usuarios }\end{array}$ & 140 & 3 & 5 & 4,76 & , 478 \\
\hline $\begin{array}{l}\text { Profesor adapta las clases a las } \\
\text { necesidades de los usuarios }\end{array}$ & 140 & 3 & 5 & 4,71 &, 540 \\
\hline $\begin{array}{l}\text { El profesor anima } \\
\text { suficientemente al grupo }\end{array}$ & 140 & 3 & 5 & 4,74 &, 517 \\
\hline $\begin{array}{l}\text { El profesor tiene las clases bien } \\
\text { planificadas }\end{array}$ & 140 & 3 & 5 & 4,78 & ,481 \\
\hline$N$ válido (por lista) & 140 & & & & \\
\hline
\end{tabular}

Leyenda: N-Número de muestra; S- Desviación estándar; $\bar{X}$-Media aritmética

Nota: Adaptación de dimensiones de la percepción (Nuviala et al, 2008, p. 11). Elaboración propia en SPSS 24

En la dimensión instalaciones y material, se continúa observando una satisfacción muy alta de todos los usuarios $(\bar{X}=4,59)$ (Tabla 9). Particularidad definida: tres de los ocho ítems recibieron la más alta calificación con una $\bar{X}=4,69$. Estos son: se dispone de suficiente material para las clases, el material está en condiciones óptimas para su uso, la seguridad de la instalación es la adecuada y a su vez con un puntaje menos valorado pero positivo, el material es moderno, con un puntaje de 4,49 (Tabla 10).
Tabla 9. Estadístico descriptivo

\begin{tabular}{|c|c|c|c|c|c|}
\hline \multirow{2}{*}{ Estadístico descriptivo } & \multirow{2}{*}{$\mathbf{N}$} & \multicolumn{2}{|c|}{ Valores } & \multirow{2}{*}{$\bar{X}$} & \multirow{2}{*}{$\mathbf{s}$} \\
\hline & & Mínimo & Máximo & & \\
\hline $\begin{array}{l}\text { Dimensión Instalaciones } \\
\text { y Material }\end{array}$ & 140 & 2,38 & 5,00 & 4,5973 & 0,44329 \\
\hline$N$ válido (por lista) & 140 & & & & \\
\hline
\end{tabular}

Leyenda: N-Número de muestra; S- Desviación estándar; $\bar{X}_{\text {-Media }}$ aritmética

Nota: Adaptación de dimensiones de la percepción (Nuviala et al, 2008, pp. 11). - Elaboración propia en SPSS 24

Tabla 10. Dimensión Instalaciones y Material

\begin{tabular}{|c|c|c|c|c|c|}
\hline \multirow{2}{*}{$\begin{array}{c}\text { Dimensión Instalaciones y } \\
\text { Material }\end{array}$} & \multirow{2}{*}{$\mathbf{N}$} & \multicolumn{2}{|c|}{ Valores } & \multirow{2}{*}{$\bar{X}$} & \multirow{2}{*}{$\mathbf{s}$} \\
\hline & & Mínimo & Máximo & & \\
\hline $\begin{array}{l}\text { Los vestuarios están } \\
\text { adecuadamente limpios }\end{array}$ & 140 & 1 & 5 & 4,55 & 0,723 \\
\hline $\begin{array}{l}\text { Los vestuarios son } \\
\text { adecuadamente amplios }\end{array}$ & 140 & 1 & 5 & 4,56 & 0,722 \\
\hline $\begin{array}{l}\text { Las instalaciones están } \\
\text { adecuadamente amplias }\end{array}$ & 140 & 2 & 5 & 4,59 & 0,667 \\
\hline La temperatura es la apropiada & 140 & 1 & 5 & 4,53 & 0,753 \\
\hline $\begin{array}{l}\text { Se dispone de suficiente } \\
\text { material para las clases }\end{array}$ & 140 & 3 & 5 & 4,69 & 0,576 \\
\hline $\begin{array}{l}\text { El material está en condiciones } \\
\text { óptimas para su uso }\end{array}$ & 140 & 2 & 5 & 4,69 & 0,564 \\
\hline El material es moderno & 140 & 2 & 5 & 4,49 & 0,704 \\
\hline $\begin{array}{l}\text { La seguridad de la instalación } \\
\text { es la adecuada }\end{array}$ & 140 & 1 & 5 & 4,69 & 0,599 \\
\hline N válido (por lista) & 140 & & & & \\
\hline
\end{tabular}

Leyenda: N-Número de muestra; S- Desviación estándar; $\bar{X}$-Media aritmética

Nota: Adaptación de dimensiones de la percepción (Nuviala et al, 2008, pp. 11). - Elaboración propia en SPSS 24

Continuando con la dimensión actividades los usuarios perciben que los diferentes trabajos a realizar en cada sesión de entrenamiento son muy buenos, por lo tanto, obtuvieron una $\bar{X}=4,60$ como calificación general (Tabla 11); estando en primer lugar con una puntuación de 4,76 la actividad es amena (agradable) y con una menor puntuación, aunque bien calificada el ítem, está informado/a sobre los beneficios de esta actividad con una $\bar{X}=4,41$ (Tabla 12 ). 
Tabla 12. Estadístico descriptivo

\begin{tabular}{cccccc}
\hline \multirow{2}{*}{ Estadístico descriptivo } & \multirow{2}{*}{$\mathbf{N}$} & \multicolumn{2}{c}{ Valores } & $\overline{\text { Mínimo Máximo }}$ & \multirow{2}{*}{$\mathbf{S}$} \\
\hline Dimensión actividades & 140 & 3,38 & 5,00 & $4,6054,42681$ \\
$N$ válido (por lista) & 140 & & & \\
\hline
\end{tabular}

Leyenda: N-Número de muestra; S- Desviación estándar; $\bar{X}_{\text {-Media }}$ aritmética

Nota: Adaptación de dimensiones de la percepción (Nuviala et al, 2008, pp. 11). - Elaboración propia en SPSS 24

Tabla 11. Dimensión Actividades

\begin{tabular}{lcccccc}
\hline \multicolumn{1}{c}{ Dimensión Actividades } & $\mathbf{N}$ & \multicolumn{3}{c}{ Valores } & & S \\
\cline { 3 - 4 } & & \multicolumn{3}{c}{ Mínimo Máximo } & $X$ & \\
\hline $\begin{array}{l}\text { La oferta de actividades se } \\
\text { renueva }\end{array}$ & 140 & 1 & 5 & 4,52 &, 694 \\
$\begin{array}{l}\text { La actividad es amena. } \\
\text { (agradable) }\end{array}$ & 140 & 3 & 5 & 4,76 &, 503 \\
$\begin{array}{l}\text { Las tareas que desarrollan en } \\
\text { clase son variadas }\end{array}$ & 140 & 1 & 5 & 4,60 &, 718 \\
$\begin{array}{l}\text { Los horarios son adecuados para } \\
\text { los usuarios }\end{array}$ & 140 & 2 & 5 & 4,63 &, 639 \\
$\begin{array}{l}\text { Las actividades terminan en el } \\
\text { tiempo indicado }\end{array}$ & 140 & 1 & 5 & 4,66 &, 643 \\
$\begin{array}{l}\text { Está informado/a sobre los } \\
\text { beneficios de esta actividad }\end{array}$ & 140 & 1 & 5 & 4,41 &, 906 \\
$\begin{array}{l}\text { Está contento/a con la relación } \\
\text { calidad/precio de la actividad }\end{array}$ & 140 & 2 & 5 & 4,64 &, 612 \\
$\begin{array}{l}\text { Con esta actividad consigo los } \\
\text { resultados que esperaba }\end{array}$ & 140 & 3 & 5 & 4,61 &, 570 \\
\begin{tabular}{l}
$N$ válido (por lista) \\
\hline
\end{tabular} & 140 & & & & \\
\hline
\end{tabular}

Leyenda: N-Número de muestra; S- Desviación estándar; $\bar{X}_{\text {-Media }}$ aritmética

Nota: Adaptación de dimensiones de la percepción (Nuviala et al, 2008, pp. 11). - Elaboración propia en SPSS 24

Finalizando con el análisis de las dimensiones se encuentra la imagen de la organización; de todas esta es la de más baja puntuación, aunque su calificación es positiva con una $\bar{X}=4,35$ (Tabla 13). En esta dimensión existe un ítem donde la puntuación de la satisfacción es bastante baja con relación a los ítems de las dimensiones mencionadas con anterioridad con una $\bar{X}=3,33$ el cual es, medios para transmitir información (Buzón de sugerencias) y de mejor percepción para el usuario es, hay buena relación entre el personal de la instalación con una puntuación de 4,77 (Tabla 14).
Tabla 14. Estadístico descriptivo

\begin{tabular}{cccccc}
\hline Estadístico descriptivo & \multirow{N}{*}{$\mathbf{N}$} & \multicolumn{2}{c}{ Valores } & \multirow{X}{*}{$\mathbf{S}$} & S \\
\cline { 3 - 5 } & & Mínimo & Máximo & & \\
\hline $\begin{array}{c}\text { Dimensión Imagen de la } \\
\text { Organización }\end{array}$ & 140 & 2,67 & 5,00 & $4,3595,52220$ \\
$N$ válido (por lista) & 140 & & & \\
\hline
\end{tabular}

Leyenda: N-Número de muestra; S- Desviación estándar; $\bar{X}_{\text {-Media }}$ aritmética

Nota: Adaptación de dimensiones de la percepción (Nuviala et al, 2008, pp. 11). - Elaboración propia en SPSS 24

Tabla 13. Dimensión Imagen de la Organización

\begin{tabular}{|c|c|c|c|c|c|}
\hline \multirow{2}{*}{$\begin{array}{l}\text { Dimensión Imagen de la } \\
\text { Organización }\end{array}$} & \multirow{2}{*}{$\mathrm{N}$} & \multicolumn{2}{|c|}{ Valores } & \multirow{2}{*}{$\bar{X}$} & \multirow{2}{*}{$\mathrm{S}$} \\
\hline & & Mínimo & Máximo & & \\
\hline $\begin{array}{l}\text { Medios para transmitir } \\
\text { información. (buzón de } \\
\text { sugerencias) }\end{array}$ & 140 & 1 & 5 & 3,33 & 1,354 \\
\hline $\begin{array}{l}\text { La información sobre las } \\
\text { actividades en el centro es } \\
\text { adecuada }\end{array}$ & 140 & 1 & 5 & 4,22 & ,930 \\
\hline $\begin{array}{l}\text { Le ha resultado fácil integrarse } \\
\text { en la actividad }\end{array}$ & 140 & 2 & 5 & 4,56 & 722 \\
\hline $\begin{array}{l}\text { El personal de servicios está } \\
\text { cuando se le necesita }\end{array}$ & 140 & 1 & 5 & 4,57 & ,711 \\
\hline $\begin{array}{l}\text { I trato del personal de la } \\
\text { nstalación es agradable }\end{array}$ & 140 & 1 & 5 & 4,71 & ,606 \\
\hline $\begin{array}{l}\text { Hay buena relación entre el } \\
\text { personal de la instalación }\end{array}$ & 140 & 3 & 5 & 4,77 & 438 \\
\hline$N$ válido (por lista) & 140 & & & & \\
\hline
\end{tabular}

\section{DISCUSIÓN}

Si lo que se quiere es mejorar el servicio de la organización, es indiscutible saber acerca de la valoración que hacen los usuarios sobre las dimensiones que conforman la organización y los servicios que presta.

Comenzando con la dimensión técnico se observa que es la puntuación mas alta de todas las dimensiones. Al conocer varios trabajos realizados por (Nuviala y Casajús, 2005; Nuviala y cols, 2008; Nuviala y cols, 2012; Inderenvigado, 2015) era un resultado de esperarse. Según Nuviala Nuviala et al (2008): "... los recursos humanos directos son uno 
de los puntos más fuertes de los servicios deportivos". La elección del personal fue la adecuada. La calificación de los items en esta dimensión es muy alta, estando la de menor puntuación, el profesor adapta las clases a las necesidades de los usuarios, por encima de cuatro.

En la dimensión instalaciones $y$ material, también se obtuvo una valoración positiva muy alta, lo que no ocurre en el trabajo realizado por (Nuviala y cols, 2008) con un resultado por debajo de cuatro y el desarrollado por (Inderenvigado, 2015) con un valor por debajo de $50 \%$. En esta ocasión los ítems están calificados por encima de cuatro. La calificación de los items no varian: los usurios estan satisfechos con el aseo, la seguridad y la amplitud de las instalaciones. En lo que se refiere a los materiales, los usuarios se encuentran por igual satisfechos, por lo nuevo que están estos y el cuidado que se les presta.

Siguiendo con la dimensión actividades también se encuentra una valoración bastante alta, que coincide con los estudios de (Nuviala y Casajús, 2005; Nuviala y Cols, 2008; Nuviala y cols, 2012; Inderenvigado, 2015), donde los resultados obtenidos fueron positivos. Relacionando la satisfacción de los usuarios con el servicio que se le presta a sus hijos, se puede decir que los usuarios se identifican satisfactoriamente con las actividades efectuadas en las diferentes horas de entrenamiento. El aspecto con la menor puntuación es, esta informado/a sobre los beneficios de esta actividad. Este resultado ha sorprendido por el hecho de que los padres de familia están directamente involucrados en las actividades a trabajar.

La última dimensión trabajada fue la imagen de la organización, la cual obtuvo la valoración más baja de las cuatro; alcanzó una valoración media de 4,35 sobre 5 , siendo unos resultados similares a otros estudios, incluso aparecen cifras menores en (Nuviala y cols, 2008; Nuviala y Cols, 2012). El item donde se encontró descontento en los usuarios fue medios para transmitir información. (Buzón de sugerencias) con una $\bar{X}=3,33$. Este resultado es lógico porque la organización no cuenta con un buzón de sugerencias o tablón de anuncios, un campo en el que la organización deportiva debe trabajar, al deber ser la comunicación un elemento fundamental en toda institución.

\section{CONCLUSIONES}

El proyecto de iniciación deportiva Cantera de Héroes del Envigado FC, ha logrado producir una satisfacción general en sus clientes, ha facilitado el incremento en el número de inscritos, pues las experiencias positivas de los padres de familia se convirtieron en el principal elemento publicitario para el servicio deportivo, comprobando una vez más que el "voz a voz" es una estrategia de marketing económica y de gran impacto en las intenciones de compra de nuevos usuarios.

Es evidenciable que los aciertos de los administrativos del proyecto en la elección de los entrenadores han sido fundamentales para el éxito de la propuesta, dado que, los aspectos valorados en los técnicos y las actividades que estos desarrollan en cada una de las sesiones deportivas fueron los de mejor calificación por parte de los clientes, convirtiéndose en un punto importante para el diseño de nuevos programas de iniciación deportiva en primera infancia. Las instalaciones y el material que se utilizaron para el desarrollo de las sesiones de trabajo en el proyecto, igualmente presentaron una buena apreciación por parte de los encuestados, aunque, es importante tener presente que son necesarias la actualización y la remodelación periódica de dichos elementos para que no desciendan los niveles de satisfacción alcanzados. 
Finalmente, es importante recomendar a los administradores del proyecto el diseño de un plan de comunicaciones que facilite la circulación de la información en la que se utilicen diversos canales, y de esta manera se posibilite la realimentación constante de las dinámicas del centro de iniciación deportiva, teniendo en cuenta que, la falta de respuesta o información pertinente puede ser un factor que amenace claramente la estabilidad y consolidación de este.

\section{REFERENCIAS}

Arocas , R.L., y Mundina , J. (1998). La satisfacción del consumidor en el marketing del deporte .[ Revista de Psicología del Deporte] , 149-158

Benjamindespensa. (S.d de S.m de 2007). Benjamindespensa.tripod. com. Obtenido de http://www. benjamindespensa.tripod.com

Crespo , J. y Pérez-Campos. (2011). La influencia de la satisfacción con la programación de partidos en la intención de asistir de los espectadores. [Journal of sports economics, 48-54]

Elasri, A., Triadó, X. y Aparicio, P. (2015). La satisfacción de los clientes de los centros deportivos municipales de barcelona.[ Apunts. Educación física y deportes], 109-117

Elasri, A., Triadó, X. y Aparicio, P. (2013). Evolución de los factores de satisfacción de clientes en centros deportivos entre 1996 y 2013. ¿Cómo han cambiado las percepciones de los usuarios? (s.n), 1-17

García, J., Cepeda, G. y Martín, D. (2012). La satisfacción de clientes y su relación con la percepción de calidad en centro de fitness: utilización de la escala calidfit.[ Revista de Psicología del Deporte], 309-319
Henao, C.F., y Vargas, J.C. (2008). Evaluación de la satisfacción de los clientes de la liga antioqueña de fútbol con respecto a los programas o servicios. Viref, 1-144

Inderenvigado. (S.d de S.m de 2015). Inderenvigado.gov.co. Obtenido de https://www.inderenvigado.gov.co

Martínez , J.A. y Martínez, L. (2010). La medición de la satisfacción del consumidor de servicios deportivos a través de la lógica borrosa. [ Revista de Psicología del Deporte], 41-58

Nuviala, A. y Casajús, J.A. (2005). Calidad percibida del servicio deportivo en edad escolar desde la perspectiva de los padres. El caso de la provincia de Huelva. Internacional de Medicina y Ciencias de la Actividad Física y el Deporte, 1-12

Nuviala , A., Pérez-Ordás , R., Boceta, M., Grao-Cruces, A., Nuviala , R. y González, J. A. (2012). Calidad, satisfacción y valor percibido de los usuarios de un servicio deportivo público. Artigos originais , 11-32

Nuviala, A., Tamayo, J.A., Iranzo Llopis , J. y Falcón, D. (2008). Creación, diseño, validación y puesta en práctica de un instrumento de medición de la satisfacción de usuarios de organizaciones que prestan servicios deportivos. Retos. Nuevas tendencias en educación física, deporte y recreación, 10-16. 


\section{Anexo 1}

Encuesta de satisfacción del usuario

Al realizar esta encuesta buscamos determinar el nivel de satisfacción de nuestros usuarios con relación a los servicios prestados por el proyec "Cantera de Héroes Envigado Fútbol Club".

Nota: diligenciar esta encuesta no le tomará más de cinco (5) minutos.

Esta encuesta va dirigida hacia los padres o acompañantes y la información que aparece en esta se llena con los datos del encuestado. Marque con una $(\mathrm{X})$ el ítem que más se ajuste a su opinión
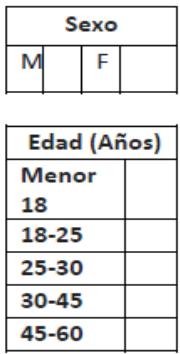

\begin{tabular}{|l|l|}
\hline \multicolumn{2}{|c|}{ Residencia } \\
\hline Medellín & \\
\hline Bello & \\
\hline La Estrella & \\
\hline Caldas & \\
\hline Sabaneta & \\
\hline Copacabana & \\
\hline Envigado & \\
\hline Itagüí & \\
\hline Otro: & \\
\hline
\end{tabular}

\begin{tabular}{|l|l|}
\hline \multicolumn{2}{|c|}{ Nivel Educativo } \\
\hline Primaria & \\
\hline Bachiller & \\
\hline Técnico & \\
\hline Tecnólogo & \\
\hline Profesional & \\
\hline Postgrado & \\
\hline
\end{tabular}

\begin{tabular}{|l|l|}
\hline \multicolumn{2}{|c|}{$\begin{array}{c}\text { Año de } \\
\text { ingreso al } \\
\text { programa }\end{array}$} \\
\hline 2014 & \\
\hline 2015 & \\
\hline 2016 & \\
\hline
\end{tabular}

\begin{tabular}{|l|l|}
\hline \multicolumn{2}{|l|}{$\begin{array}{l}\text { Horario de } \\
\text { entrenamiento }\end{array}$} \\
\hline $8-9$ am & \\
\hline $9-10$ am & \\
\hline $10-11$ am & \\
\hline $11-12$ am & \\
\hline $5-7 \mathrm{pm}$ & \\
\hline
\end{tabular}

\begin{tabular}{|l|l|}
\hline \multicolumn{2}{|c|}{ Estado civil } \\
\hline Soltero/a & \\
\hline Casado/a & \\
\hline Divorciado/a & \\
\hline Viudo/a & \\
\hline Unión libre & \\
\hline Separado/a & \\
\hline
\end{tabular}

Marque con una $\mathbf{X}$ el ítem con el cual se identifique siendo 1(muy en desacuerdo, el más bajo) y 5 (muy de acuerdo, el más alto).

\begin{tabular}{|c|c|c|c|c|c|}
\hline \multicolumn{6}{|l|}{ Dimensiones de la percepción } \\
\hline Técnicos & $\begin{array}{c}\text { Muy en } \\
\text { desacuerdo }\end{array}$ & $\begin{array}{c}\text { En } \\
\text { desacuerdo }\end{array}$ & $\begin{array}{c}\text { De } \\
\text { acuerdo }\end{array}$ & $\begin{array}{c}\text { Bastante } \\
\text { de acuerdo }\end{array}$ & $\begin{array}{l}\text { Muy de } \\
\text { acuerdo }\end{array}$ \\
\hline El profesor es respetuoso. & 1 & 2 & 3 & 4 & 5 \\
\hline $\begin{array}{l}\text { Está contento/a con el trato dispensado por los } \\
\text { profesores. }\end{array}$ & 1 & 2 & 3 & 4 & 5 \\
\hline $\begin{array}{l}\text { Cree que presta el profesor una atención adecuada } \\
\text { a los usuarios-alumnos desde el primer día. }\end{array}$ & 1 & 2 & 3 & 4 & 5 \\
\hline $\begin{array}{l}\text { Cree que el profesor adapta las clases a los } \\
\text { intereses-necesidades de los alumnos-usuarios. }\end{array}$ & 1 & 2 & 3 & 4 & 5 \\
\hline $\begin{array}{l}\text { Considera que el profesor anima suficientemente al } \\
\text { grupo. }\end{array}$ & 1 & 2 & 3 & 4 & 5 \\
\hline $\begin{array}{l}\text { Percibe que el profesor tiene las clases bien } \\
\text { planificadas. }\end{array}$ & 1 & 2 & 3 & 4 & 5 \\
\hline \multicolumn{6}{|l|}{ Instalaciones y material } \\
\hline Los vestuarios están suficientemente limpios. & 1 & 2 & 3 & 4 & 5 \\
\hline Los vestuarios son los suficientemente amplios. & 1 & 2 & 3 & 4 & 5 \\
\hline Las instalaciones están suficientemente limpias. & 1 & 2 & 3 & 4 & 5 \\
\hline La temperatura es la adecuada. & 1 & 2 & 3 & 4 & 5 \\
\hline Se dispone de suficiente material para las clases. & 1 & 2 & 3 & 4 & 5 \\
\hline El material está en condiciones óptimas para su uso. & 1 & 2 & 3 & 4 & 5 \\
\hline El material es moderno. & 1 & 2 & 3 & 4 & 5 \\
\hline La seguridad de la instalación es la adecuada. & 1 & 2 & 3 & 4 & 5 \\
\hline \multicolumn{6}{|l|}{ Actividades } \\
\hline La oferta de actividades se actualiza. & 1 & 2 & 3 & 4 & 5 \\
\hline La actividad es amena. (agradable) & 1 & 2 & 3 & 4 & 5 \\
\hline $\begin{array}{l}\text { Las tareas que desarrolla en la clase son lo } \\
\text { suficientemente variadas. }\end{array}$ & 1 & 2 & 3 & 4 & 5 \\
\hline Los horarios son convenientes para los usuarios. & 1 & 2 & 3 & 4 & 5 \\
\hline Las actividades finalizan en el tiempo indicado. & 1 & 2 & 3 & 4 & 5 \\
\hline $\begin{array}{l}\text { Está informado/a sobre los beneficios de esta } \\
\text { actividad. }\end{array}$ & 1 & 2 & 3 & 4 & 5 \\
\hline $\begin{array}{l}\text { Está satisfecho/a con la relación calidad/precio de la } \\
\text { actividad. }\end{array}$ & 1 & 2 & 3 & 4 & 5 \\
\hline $\begin{array}{l}\text { Con esta actividad obtengo los resultados que } \\
\text { esperaba. }\end{array}$ & 1 & 2 & 3 & 4 & 5 \\
\hline \multicolumn{6}{|l|}{ IMAGEN DE LA ORGANIZACIÓN } \\
\hline $\begin{array}{l}\text { Disponen las instalaciones de algún medio para } \\
\text { transmitir sus sugerencias (buzón de sugerencias, } \\
\text { tablón d anuncios). }\end{array}$ & 1 & 2 & 3 & 4 & 5 \\
\hline $\begin{array}{l}\text { La información sobre las actividades que se } \\
\text { desarrollan en el centro es adecuada. }\end{array}$ & 1 & 2 & 3 & 4 & 5 \\
\hline $\begin{array}{l}\text { Le ha resultado sencillo incorporarse en la actividad } \\
\text { que participa. }\end{array}$ & 1 & 2 & 3 & 4 & 5 \\
\hline $\begin{array}{l}\text { El personal de servicio está cuando se le necesita y } \\
\text { siempre está dispuesto a ayudarle. }\end{array}$ & 1 & 2 & 3 & 4 & 5 \\
\hline El trato del personal de la instalación es agradable. & 1 & 2 & 3 & 4 & 5 \\
\hline $\begin{array}{l}\text { Hay buena relación entre el personal de la } \\
\text { instalación. }\end{array}$ & 1 & 2 & 3 & 4 & 5 \\
\hline
\end{tabular}

